\title{
Catheter-based delineation of lumpectomy cavity for accurate target definition in partial-breast irradiation with multicatheter interstitial brachytherapy
}

\author{
Kazuhiko Sato, MD, PhD', Takahiro Shimo, MS², Hiromi Fuchikami, MD!, Naoko Takeda, MD!', Masahiro Kato, MD, PhD², \\ Tomohiko Okawa, MD, $\mathrm{PhD}^{3}$ \\ 'Department of Breast Oncology, Tokyo-West Tokushukai Hospital, Matsubara, Akishima, Tokyo, Japan, ${ }^{2}$ Department of Radiation Oncology, \\ Tokyo-West Tokushukai Hospital, Matsubara, Akishima, Tokyo, Japan, ${ }^{3}$ Preventive Medical Center, Sano City Hospital, Tanuma, Sano, Tochigi, \\ Japan
}

\begin{abstract}
Purpose: In partial-breast irradiation (PBI), accurate lumpectomy cavity (LC) delineation is critical. Seroma-based delineation (SBD) using computed tomography (CT) with clips remains uncertain, causing an expansion of the LC and planning target volume (PTV). In catheter-based delineation (CBD), the implanted catheters were used as reference markers for LC delineation in multicatheter interstitial brachytherapy (MIB).

Material and methods: Between October 2008 and October 2018, 513 patients who underwent MIB-PBI were examined. In CBD, anatomical relations of LC to catheters were recorded. In randomly selected 22 CBD cases, the LC volume and PTV were retrospectively recontoured on SBD, and the relationship between the contribution of CBD and cavity visuality was evaluated. The LC volume and PTV before and after the introduction of CBD were compared.

Results: The mean LC volumes based on SBD and CBD were $19.1 \mathrm{~cm}^{3}$ and $14.1 \mathrm{~cm}^{3}$, respectively $(p<0.001)$. The mean PTVs based on SBD and CBD were $47.9 \mathrm{~cm}^{3}$ and $35.7 \mathrm{~cm}^{3}$, respectively $(p<0.0001)$. More reductions in the LC volume $\left(5.1 \mathrm{~cm}^{3}\right)(p<0.05)$ and PTV $\left(7.7 \mathrm{~cm}^{3}\right)(p=0.13)$ were observed in the poorly visible LC than in the visible LC. The LC volume and PTV before the introduction of CBD $(n=411)$ were compared with those after introduction $(n=102)$. Significant reductions were observed in the LC volume $\left(5.9 \mathrm{~cm}^{3}\right)(p<0.0001)$ after the introduction of CBD; moreover, PTV tended to be reduced $\left(3.9 \mathrm{~cm}^{3}\right)(p=0.17)$.

Conclusions: CBD may help to establish the standardized procedure for MIB-PBI and prevent unnecessary radiation exposure to the normal breast tissue.

J Contemp Brachytherapy 2019; 11, 2: 108-115 DOI: https://doi.org/10.5114/jcb.2019.84504
\end{abstract}

Key words: breast cancer, partial-breast irradiation, multicatheter interstitial brachytherapy, delineation, lumpectomy cavity.

\section{Purpose}

Breast-conserving therapy (BCT) comprising breastconserving surgery (BCS) and whole-breast irradiation (WBI) has been recognized as a standard local therapy for patients with early-stage breast cancer. Recently, this treatment has been reported to not only maintain the body image, but also have a possible benefit of better overall survival than mastectomy [1,2]. However, some patients choose mastectomy over BCT, even with favorable features of local recurrence due to limited access to radiation facilities and potential side effects of radiotherapy [3]. Evidence on partial-breast irradiation (PBI) as an alternative radiotherapy to WBI to solve the aforementioned issues has been accumulated. Among the several PBI techniques reported, there are two techniques with high-level evidence: 5/6-day multicatheter interstitial brachytherapy (MIB) by the Groupe Européen de Curiethérapie (GEC) and the European Society for Radiotherapy \& Oncology (ESTRO) trial [4,5], and 3-week intensity-modulated radiotherapy (IMRT) by the Intensity Modulated and Partial Organ Radiotherapy (IMPORT) LOW trial [6], showing equivalent tumor control and toxicities. In PBI with highdose per fraction, the amount of radiation should be controlled to prevent late toxicity and poor cosmetic outcome 
$[7,8]$. In PBI treatment via brachytherapy, the planning target volume (PTV) can be reduced compared with external radiation beam techniques because of the lack of setup error and respiratory movement, and MIB-PBI has potential benefits by improving cosmesis and quality of life $[9,10]$.

To minimize PTV in MIB-PBI with satisfactory tumor control and cosmetic outcomes, accurate catheter placement and lumpectomy cavity (LC) delineation are critical. We performed MIB-PBI using the intraoperative open-cavity implant (IOCI) technique, which showed outcomes equivalent to those in other western countries $[11,12]$. In target planning, referring to preoperative and post-operative computed tomography (CT) images and a surgical clip placement could help in accurate LC delineation $[13,14]$. Although the IOCI technique has an advantage of accurate catheter insertion into the tumor bed, several problems of CT/clip-assisted seroma-based delineation (SBD) remain, including migrating clips, a limited number of clips for irregular LC shapes, and invisible fluid-filled cavity in dense breasts [15]. Uncertainties in delineating LC can be obviated by adding margins, leading to an increase in dose to the normal tissue. This radiation field expansion with uncertainty has a harmful influence on small-breasted patients. Therefore, a definite LC delineation method to perform MIB-PBI is particularly needed in introducing the western technique in Asian women because of their small breast size [16].

In performing MIB-PBI, implanted catheters provide additional geographic information of the LC on CT/clip image, which could be used as reference markers. We developed the catheter-based delineation (CBD) technique to contour LC more accurately, leading to a reduction of unnecessary radiation dose to the normal tissue. This study aimed to evaluate the importance of additional information obtained from CBD and SBD in reducing LC volume and PTV.

\section{Material and methods}

\section{Patient and breast-conserving surgery}

Between October 2008 and October 2018, 513 consecutive patients who underwent BCS followed by MIB-PBI using the IOCI technique were examined. In general, our MCB-PBI treatment has been employed for patients satisfying the following criteria: aged $\geq 40$ years, tumor diameter $\leq 3 \mathrm{~cm}$, pN0-1 mi, and negative surgical margin, which were similar to the inclusion criteria of the GEC-ESTRO trial [4]. In our study, BCS was a lumpectomy performed as per the American standard to excise a tumor with minimum 1-cm gross rim of the adjacent tissue with deep-layer approximation [17]. Using a specimen mammography, additional tissue was resected if the margin appeared close to the tumor. After subcutaneous skin closure, the LC was filled with the standard saline to attempt breast contour. This procedure is our routine practice with or without an attempt of catheter implant.

\section{Catheter implant and radiation planning}

The basic IOCI technique has been previously introduced [18]. Before surgery, a contrast-enhanced CT was obtained to determine the implant geometry using the PLATO (Version 14.3.5, Nucletron, an Elekta company, Stockholm, Sweden) or the Oncentra (Version 4.5.1.71, Nucletron) treatment planning system. Sentinel nodes were confirmed to be negative for metastasis before catheter implant. Four surgical metallic clips were placed into the tumor bed to mark the extent of the cavity. Referring to the implant simulation on preoperative $\mathrm{CT}$, all entry and exit points of catheters were marked on the skin surface using a free-hand template, which were spaced to form equilateral triangles of 1.0 or $1.6 \mathrm{~cm}$. The stainless-steel rigid needles were manually inserted into the LC from the surface to the depth. The flexible plastic catheters that are used for introducing iridium wires were replaced and attached with buttons at the ends.

Immediately after surgery, non-contrast CT (Aquilion 64; Canon Medical Systems, Tochigi, Japan) images of slices with 2-mm thickness were obtained to identify the cavity with the seroma and surgical clips. The data were transferred to the radiotherapy planning system. The LC with high visibility was delineated with the homogeneous water density area, excluding protrusions or sharp irregularities and clips. When visualization of the accumulated fluid is difficult, the LC included retained water area, clips, and surrounding area to be considered, and the resection cavity with preoperative CT images as a reference [19]. The PTV equals the clinical target volume, including the LC after volume expansion of $1.0-\mathrm{cm}$ around the cavity, and edited by limiting the skin by $0.5 \mathrm{~cm}$ and up to the pectoral muscle, which was considered as PTV as per the American Brachytherapy Society (ABS) and the National Surgical Adjuvant Breast and Bowel Project (NSABP) B-39/Radiation Therapy Oncology Group (RTOG) 0413 protocol [20]. The basal dose points were defined in the central axis according to the Paris system, and the reference dose prescription of $85 \%$ was chosen. The first planning priority was to cover minimum $90 \%$ of the PTV, with $90 \%$ of the prescription dose. The actual volumes of tissues receiving $150 \%\left(\mathrm{~V}_{150}\right)$ and $200 \%\left(\mathrm{~V}_{200}\right)$ of the prescribed dose were also limited to $\leq 70 \mathrm{~cm}^{3}$ and $\leq 20 \mathrm{~cm}^{3}$, respectively. The maximum dose to the skin and chest wall/ rib at $<75 \%$ of the prescribed dose were attempted. The breast volume was calculated using a three-dimensional CT with anatomical boundaries in the RTOG Breast Cancer Atlas [21].

High-dose-rate interstitial brachytherapy with an iridium-192 ( ${ }^{192} \mathrm{Ir}$ ) remote afterloading equipment was performed in an accelerated manner, with a dose of $32 \mathrm{~Gy}$ in eight fractions over 5-6 days. Each radiotherapy was delivered daily, with 6 hours or longer interval between the fractions. The catheters were removed immediately after the last radiotherapy.

\section{Catheter-based delineation}

In October 2017, the CBD technique was developed to delineate LC without uncertainty and more reproducibly by using the inserted catheters as the reference markers. The anatomical relations of LC to catheters were recorded in detail. First, the distance between the upper/lower catheters and upper/lower edges of the glandular tissue was measured directly after catheter implants. Second, the scalers were inserted into all catheters and drawn out 
A

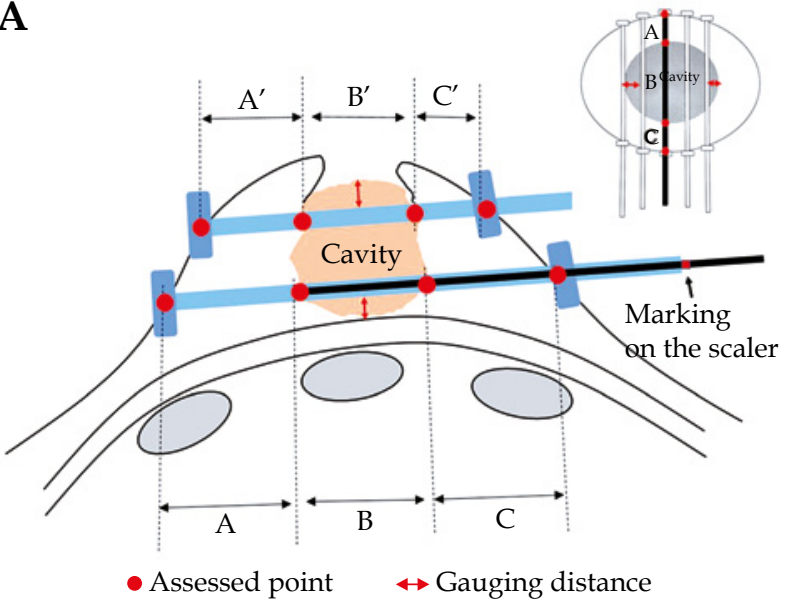

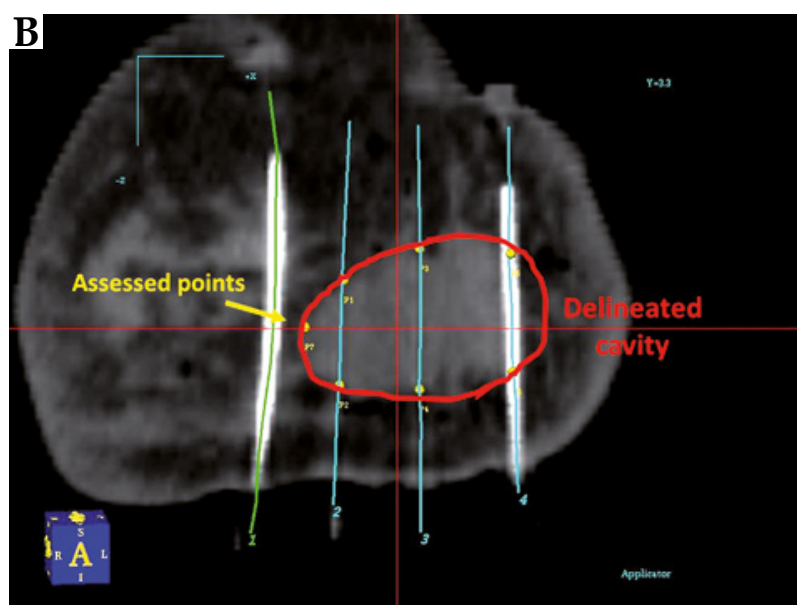

Fig. 1. A) The vertical and horizontal distances between the catheters and the glandular tissue were measured directly after placing the catheter implants (red arrow). The scalers are marked on the points representing the distance of the remaining tissues and the cavity (black arrow). B) The radiation oncologist translated the measured diameters in relation to the catheters and the cavity

to mark the following points: the scaler's edge facing the bottom of the catheter, the scaler's edge appearing from the wall of the cavity, the scaler's edge disappearing into the opposite wall of the cavity indicating the cavity distance, and the scaler's edge appearing from the outlet of skin. The stuck-out catheter's dimensions of the cavity to scrutinize its size were measured (Figure 1A). The above information was used to delineate LC (Figure 1B).

\section{Cavity visualization score}

In patients with CBD technique, each LC image was assigned a cavity visualization score (CVS) by a single observer (TS), indicating the degree of visibility of a LC using a five-point CVS numeric score from 1 to 5 [22]. CVS was ranked as follows: CVS 1 - cavity not visualized; CVS 2 - cavity visualized but margins indistinct; CVS 3 - cavity visualized with some distinct margins; CVS 4 cavity visualized with the majority of margins indistinct; and CVS 5 - all cavity margins clearly defined. The features influencing the visibility of a LC were examined.

\section{Influence of CBD on LC delineation and PTV}

In randomly selected patients with $\mathrm{CBD}$, the $\mathrm{LC}$ volumes and PTV in each image were retrospectively and separately recontoured using CT/clip-based SBD to compare with those by CBD regarding CVS. The observer (TS) could access the preoperative CT image obtained before the introduction of CBD but was blinded to the contouring information obtained from CBD. First, impact of CBD to reduce the LC volume and PTV regarding CVS was analyzed. Next, the LC volume and PTV before and after the introduction of CBD were compared to confirm the reduction of these volumes for assessing possible improvements in cosmetic outcomes obtained from MIB-PBI.

\section{Statistics}

The Student's unpaired $t$-test was used to analyze the differences in average continuous variables. The Krus-
kal-Wallis test was also used to compare clinical demographics associated with the four CVS. The probability $(p)$ values of $<0.05$ were considered to indicate statistical significance. The number following the symbol " \pm " indicates a standard deviation. Bilateral breast cancers treated by MBI-PBI were counted as two different patients in the analysis. Ethical approval was obtained from the respective committees; all patients provided an informed consent.

\section{Results}

\section{Patient characteristics and treatment-related demographics}

Between October 2017 and October 2018, the target planning was performed for the CBD of 102 lesions in 99 patients who underwent MIB-PBI using the IOCI technique. The mean patient age was $59.5 \pm 1.3$ years (range, 36-86 years). The mean pathological tumor diameter was $16.4 \pm 0.7 \mathrm{~mm}$ (range, 5-40 $\mathrm{mm}$ ). The mean LC volume, PTV, $\mathrm{V}_{150}$, and $\mathrm{V}_{200}$ were $12.8 \pm 0.7 \mathrm{~cm}^{3}$ (range, $\left.2.0-44.0 \mathrm{~cm}^{3}\right), 34.3 \pm 1.6 \mathrm{~cm}^{3}\left(\right.$ range, $\left.5.0-103.0 \mathrm{~cm}^{3}\right)$, $17.1 \pm 0.9 \mathrm{~cm}^{3}$ (range, $4.1-47.0 \mathrm{~cm}^{3}$ ), and $7.8 \pm 0.4 \mathrm{~cm}^{3}$ (range, $\left.2.2-24.8 \mathrm{~cm}^{3}\right)$, respectively. The coverage of the PTV was $91.8 \pm 0.4 \%$ (range, $90.0-101.4 \%$ ). The mean breast volume was $383.5 \pm 22.7 \mathrm{~cm}^{3}$ (range, 88.0-1286.0 $\mathrm{cm}^{3}$ ), and the whole-breast reference volume receiving the prescribed dose was $10.2 \pm 0.4 \%$ (range, $2.5-23.7 \%$ ). The maximum doses to the skin and chest wall were $2.6 \pm 0.0 \mathrm{~Gy}$ (range, 1.8-3.9 Gy) and $2.5 \pm 0.1 \mathrm{~Gy}$ (range, 0.7-4.5 Gy), respectively.

The mean number of needles per implant was $6.1 \pm 0.2$ (range, 3-13). Three-plane implants were used in 9 patients (8.8\%), two-plane implants in 46 patients $(45.1 \%)$, and one-plane implants in 47 patients $(46.1 \%)$. Patient characteristics and treatment-related demographics for each number of planes are presented in Table 1. Regardless of the number of planes, our planning priority of target volume coverage $\left(\mathrm{V}_{90} \geq 90 \%\right.$ of PTV) with the limited volume 
Table 1. Patient characteristics and treatment-related demographics for each number of planes

\begin{tabular}{|c|c|c|c|}
\hline Number of planes & $\begin{array}{c}\text { One-plane } \\
(n=47)\end{array}$ & $\begin{array}{l}\text { Two-plane } \\
(n=46)\end{array}$ & $\begin{array}{l}\text { Three-plane } \\
\qquad(n=9)\end{array}$ \\
\hline \multicolumn{4}{|c|}{ Patient characteristics (mean \pm standard deviation) } \\
\hline Mean age (years) & $57.3 \pm 1.9$ & $62.0 \pm 1.8$ & $57.8 \pm 3.8$ \\
\hline Mean breast size $\left(\mathrm{cm}^{3}\right)$ & $232.5 \pm 15.1$ & $455.0 \pm 22.9$ & $806.3 \pm 106.6$ \\
\hline Mean tumor diameter $(\mathrm{mm})^{*}$ & $15.1 \pm 0.8$ & $18.1 \pm 1.1$ & $14.8 \pm 3.2$ \\
\hline \multicolumn{4}{|c|}{ Median value of treatment-related parameters (range) } \\
\hline Number of catheters & $4(3-5)$ & $7(4-10)$ & $10(9-13)$ \\
\hline LC volume $\left(\mathrm{cm}^{3}\right)$ & $8.3(2.2-18.7)$ & $14.2(6.8-40.4)$ & $18.8(4.2-43.8)$ \\
\hline PTV $\left(\mathrm{cm}^{3}\right)$ & $21.5(4.6-68.0)$ & $38.0(14.9-89.3)$ & $52.6(31.7-103.4)$ \\
\hline $\mathrm{V}_{90}\left(\mathrm{~cm}^{3}\right)$ & $34.5(14.0-74.3)$ & $51.7(24.6-108.3)$ & $75.2(38.2-104.3)$ \\
\hline Target coverage $(\%)^{\star *}$ & $90.6(90.0-101.4)$ & $91.9(90.1-99.7)$ & $91.7(90.2-94.9)$ \\
\hline $\mathrm{V}_{100}\left(\mathrm{~cm}^{3}\right)$ & $28.5(11.8-63.6)$ & $45.0(19.4-94.1)$ & $64.4(33.0-89.8)$ \\
\hline $\mathrm{V}_{150}\left(\mathrm{~cm}^{3}\right)$ & $11.9(4.1-34.1)$ & $17.2(6.8-44.5)$ & $21.7(14.2-46.8)$ \\
\hline $\mathrm{V}_{200}\left(\mathrm{~cm}^{3}\right)$ & $6.1(2.2-19.5)$ & $7.0(3.2-16.1)$ & $8.1(5.5-19.7)$ \\
\hline$\overline{\mathrm{DHI}}$ & $0.58(0.46-0.68)$ & $0.63(0.45-0.74)$ & $0.64(0.41-0.72)$ \\
\hline COIN & $0.58(0.21-0.73)$ & $0.64(0.47-0.87)$ & $0.64(0.40-0.79)$ \\
\hline Maximum skin dose (Gy) & $2.8(1.8-3.9)$ & $2.5(2.0-3.9)$ & $2.3(1.8-2.7)$ \\
\hline Maximum chest wall/rib dose (Gy) & $2.8(1.2-4.5)$ & $2.4(0.9-3.9)$ & $1.6(0.7-3.7)$ \\
\hline
\end{tabular}

LC - lumpectomy cavity, PTV - planning target volume, DHI - dose homogeneity index $\left(1-V_{150} V_{100}\right)$, PTV ref - volume of the PTV receiving at least $100 \%$ of the prescribed dose, COIN - conformity index $\left(P T V_{\text {ref }} / P T V \times P T V_{\text {ref }} / V_{100}\right)$

*Pathologically reported measuring maximum extent of the tumor

**Target coverage (\%) was the percentage of $90 \%$ of the prescription dose in the PTV

of hyperdose area $\left(\mathrm{V}_{150} \leq 70 \mathrm{~cm}^{3}\right.$ and $\left.\mathrm{V}_{200} \leq 20 \mathrm{~cm}^{3}\right)$ was achieved in all patients.

\section{Factors influencing CVS}

The mean CVS assigned was $3.0 \pm 0.1$ : CVS 1 - 18 patients (17.6\%); CVS 2 - 24 patients (23.5\%); CVS 3 - 17 pa-

A

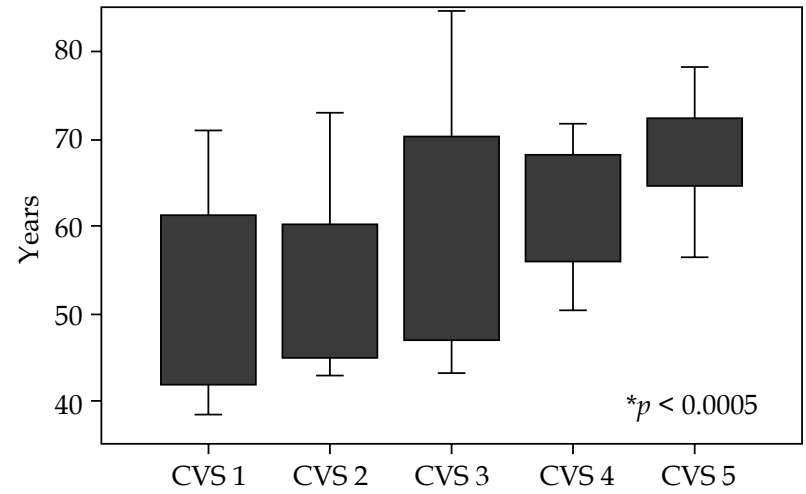

tients (16.7\%); CVS $4-22$ patients (21.6\%); and CVS 5 - 21 patients $(20.6 \%)$. The influence of clinical characteristics and contouring volume on the visibility are shown in Figures $2 \mathrm{~A}$ and $2 \mathrm{~B}$. The visibilities of the LC were significantly increased in patients with older age $(p<0.001)$ and larger LC $(p<0.05)$.

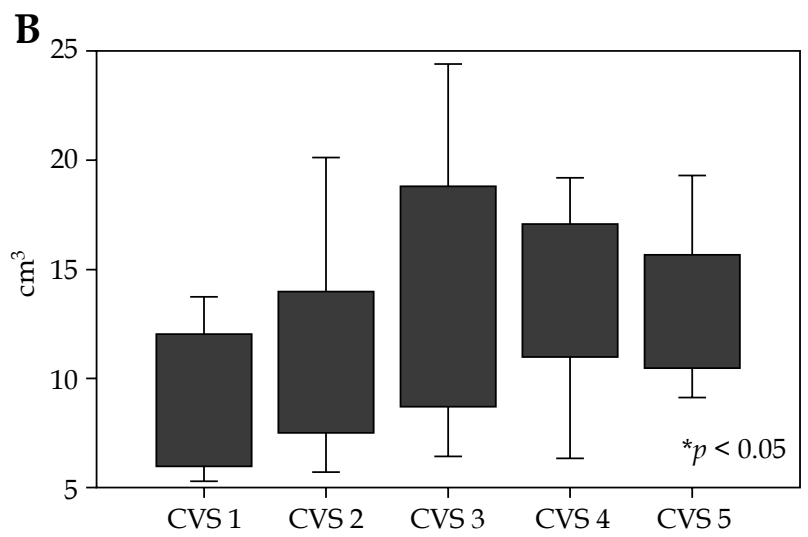

*Kruskal-Wallis test

Fig. 2. A) A box plot of the patient's age versus cavity visualization score (CVS). A significant association between the patient's age and CVS $(p=0.0002)$ was noted. The upper and lower edges of the box plot are the $75^{\text {th }}$ and $25^{\text {th }}$ percentiles. B) A box plot of the lumpectomy cavity (LC) volume versus cavity visualization score (CVS). A significant association between the LC and CVS $(p<0.05)$ was noted. The upper and lower edges of the box plot are the $75^{\text {th }}$ and $25^{\text {th }}$ percentiles 


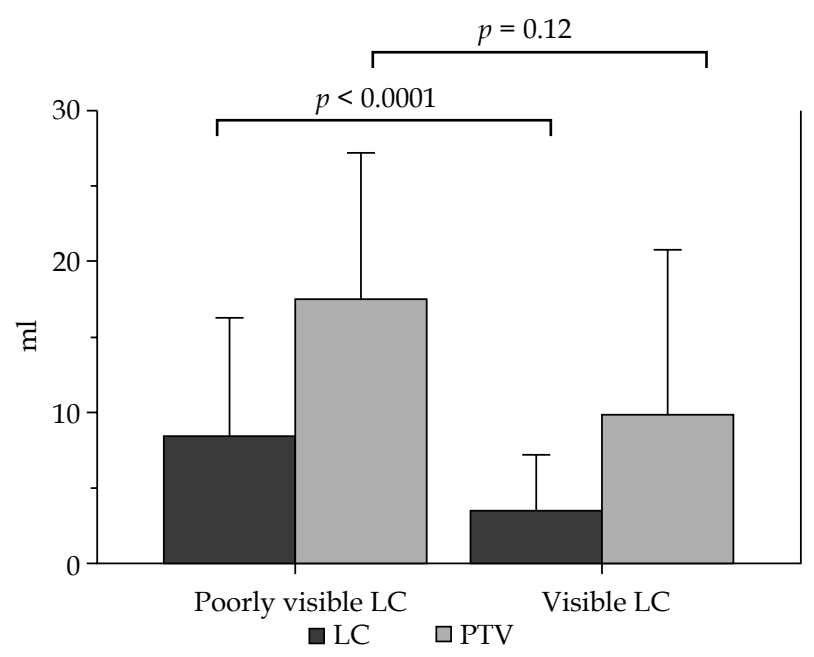

Fig. 3. Mean reduced volume of the lumpectomy cavity (LC) and planning target volume (PTV) by catheter-based delineation (CBD). A significant association between the reduced LC volume and the visibility $(p<0.0001)$ was noted, and a non-significant trend was observed between the reduced PTV and visibility $(p=0.12)$. The upper edges are the standard deviations

\section{Influence of CVS and LC volume reduction on CBD}

In 22 randomly selected patients who underwent MIB-PBI using the CBD technique [CVS $1-2$ patients (9.1\%); CVS $2-5$ patients (22.7\%); CVS $3-6$ patients (27.3\%); CVS $4-5$ patients $(22.7 \%)$; and CVS $5-4$ patients $(18.2 \%)]$, the LC volume reduction based on CBD from the recontoured LC volume by SBD was observed in 20 patients $(90.9 \%)$. The mean LC volumes based on SBD and CBD were $19.1 \pm 2.4 \mathrm{~cm}^{3}$ (range, 6.2-49.8 $\mathrm{cm}^{3}$ ) and $14.1 \pm 1.7 \mathrm{~cm}^{3}$ (range, 3.8-40.2 $\mathrm{cm}^{3}$ ), with an absolute LC volume reduction of $5.0 \pm 1.2 \mathrm{~cm}^{3}$ (range, $-1.0-24.4 \mathrm{~cm}^{3}$ ), respectively $(p<0.001)$. The mean PTV based on SBD and CBD were $47.9 \pm 4.6 \mathrm{~cm}^{3}$ (range, 13.5-97.7 $\mathrm{cm}^{3}$ ) and 35.7 $\pm 3.7 \mathrm{~cm}^{3}$ (range, 8.3-89.3 $\mathrm{cm}^{3}$ ), with an absolute PTV reduction of $12.3 \pm 2.3 \mathrm{~cm}^{3}$ (range, $-0.01-43.8 \mathrm{~cm}^{3}$ ), respectively $(p<0.0001)$. More reductions in the LC volume $\left(5.1 \mathrm{~cm}^{3}\right)\left(8.5 \pm 2.9 \mathrm{~cm}^{3}\right.$ vs. $\left.3.4 \pm 1.0 \mathrm{~cm}^{3} ; p<0.05\right)$ and PTV $\left(7.7 \mathrm{~cm}^{3}\right)\left(17.5 \pm 3.7 \mathrm{~cm}^{3}\right.$ vs. $\left.9.8 \pm 2.8 \mathrm{~cm}^{3} ; p=0.13\right)$ were observed in the poorly visible LC (CVS 1 or 2) than in the visible LC (CVS 3-5) (Figure 3). It could be considered a cause that the volume of delineated LC with poor visualization tended to include large surrounding tissue.

\section{Influence of CBD introduction to reduce the $L C$ volume and PTV}

The LC volume and PTV before the introduction of CBD $(n=411)$ were compared with those after introduction $(n=102)$. Although a slight difference in patient age $(56.9$ \pm 0.6 years vs. $59.5 \pm 1.3$ years; $p=0.06$ ) was noted, no pathological tumor diameters $\left(15.8 \pm 3.5 \mathrm{~cm}^{3}\right.$ vs. $16.4 \pm 0.7 \mathrm{~cm}^{3}$; $p=0.45$ ) between the SBD and CBD patients were reported, respectively. The significant reductions after the introduction of CBD were observed in the LC volume $\left(5.9 \mathrm{~cm}^{3}\right)\left(18.8 \pm 0.7 \mathrm{~cm}^{3}\right.$ vs. $\left.12.9 \pm 0.7 \mathrm{~cm}^{3} ; p<0.0001\right)$; moreover, PTV tended to be reduced $\left(3.9 \mathrm{~cm}^{3}\right)\left(38.2 \pm 1.4 \mathrm{~cm}^{3}\right.$ vs. $34.3 \pm 1.6 \mathrm{~cm}^{3} ; p=0.17$ ) (Table 2). It was thought to be caused by volume reduction of poorly visualized LC in the SBD patients.

\section{Discussion}

BCT is performed to achieve good tumor control, while maintaining satisfactory cosmetic result. Excision of breast with larger volume should be prevented and tumor control by BCS should be administered to achieve better cosmesis [23]. According to lesser number of lo-

Table 2. Patients' characteristics and differences in volume between two delineation techniques

\begin{tabular}{lccc} 
& \multicolumn{1}{c}{ All patients with PBI $(n=513)$} & $p$ value \\
\cline { 2 - 3 } & SBD $(n=411)$ & CBD $(n=102)$ & NS \\
\hline Mean age (years) & $56.9 \pm 0.6$ & $59.5 \pm 1.3$ & \\
\hline Age (years), $n(\%)$ & $139(33.8)$ & $26(25.5)$ & NS \\
\hline$<50$ & $218(53.1)$ & $51(50)$ & $25(24.5)$ \\
\hline $50-69$ & $54(13.1)$ & $16.4 \pm 0.7$ & \\
\hline$\geq 70$ & $15.8 \pm 0.4$ & $12(11.8)$ & $<2(80.5)$ \\
\hline Mean tumor diameter $(\mathrm{mm})^{*}$ & & $8(7.8)$ & NS
\end{tabular}

PBI - partial-breast irradiation, SBD - seroma-based delineation, CBD - catheter-based delineation, LC - lumpectomy cavity, PTV - planning target volume *Pathologically reported measuring maximum extent of the tumor 
cal recurrences after BCT by recent advances of systemic treatment, the surgical extent could be reduced to the level of no-ink-on-tumor margin [24]. In PBI, the ratio of irradiated volume to entire breast volume has been reported to be an important factor influencing the cosmetic outcomes because the volume of radiation exposure to the healthy tissue should translate to radiation fibrosis formation [25]. The NSABP/RTOG protocol establishes ideal constrains of $<60 \%$ of the whole-breast reference volume receiving $50 \%$ of the prescribed dose, and $<35 \%$ of the whole-breast reference volume receiving the prescribed dose [20]. Although the appropriate treatment margin to define the PTV from the LC remains debatable, a reduction in the unnecessary irradiated field in PBI is crucial.

In order to minimize unnecessary irradiated volume for tumor control, accurate LC delineation is critical, because uncertain LC delineation results in the increase in surgical extent, preventing marginal miss. The presence of LC on CT is more helpful for the delineation of radiotherapy target than the absence caused by the full-thickness closure of cavity [26]. However, CT imaging has limited soft tissue contrast, making it an unreliable modality in detecting small volumes of seroma for distinguishing LC from the normal glandular breast tissue, and the LC visualization was limited to patients with dense breasts (i.e., younger age) and small resection volume $[27,28]$. More accurate LC delineation to minimize the radiation target volume is required in Asian patients for good cosmesis because of their smaller breast (Table 1); however, LC was difficult to identify in Asian women due to higher breast densities than that of Caucasian women $[29,30]$. The localization of metallic clips attached to the cavity walls has been shown to reduce the risks of geographic miss [14]; however, clips defining a limited number of points for an irregular excision cavity wall surface imposed a risk of migration [31,32]. Magnetic resonance imaging providing superior soft tissue contrast may also have the potential to differentiate more clearly between the normal tissue and post-operative LC [33]. Though, an additional expensive imaging study is required for limited identification of implanted clips and few further information [34]. Therefore, the combination with CT/clip has been considered as the current gold standard.

In partial-breast brachytherapy, the timing of implant and types of catheter used are other important factors that influence accurate delineation of the minimum target volume. Although post-operative implant procedures are more frequent than the intraoperative ones, because of the lack of the final pathology report, the single-stage BCT using partial-breast brachytherapy has gradually become a popular technique to prevent the need for a second surgical procedure $[35,36,37,38,39,40,41]$. Post-operative implant can result in geographic miss due to complex cavity and seroma accumulation [42], which increases local recurrence risk when radiation field is reduced [43]. The agreement of the LC delineation between the surgeon and radiation oncologist is easier to obtain with deep-layer approximated lumpectomy than with full-thickness closure technique [44]. Most Japanese surgeons perform a "cylindrectomy" by excising tumor cylindrically with wider normal tissue [45], in which MIB-PBI could also provide excellent coverage to the entire tumor bed.

We performed MIB-PBI to small breasted patients with relatively small number of planes and catheters using CBD technique. There are two existing guidelines from the GEC-ESTRO $[19,46,47]$ and the RTOG/ABS $[20,48]$ to be referred in terms of our MIB-PBI. There were several differences between the two guidelines. While $2 \mathrm{~cm}$ of normal tissue beyond known disease extent should be treated with surgery combined with radiotherapy in the GEC-ESTRO, a uniform margin of $1 \mathrm{~cm}$ beyond the cavity should be irradiated as PTV in the RTOG/ABS. Although the target coverage of the RTOG/ABS guidelines $\left(\mathrm{V}_{90} \geq 90 \%\right.$ of PTV) is more lenient than that of the GEC-ESTRO $\left(\mathrm{V}_{100} \geq 90 \%\right.$ of PTV), the first planning priority in our protocol $\left(\mathrm{V}_{90} \geq\right.$ $90 \%$ of PTV) has been achieves in all patients. The dose uniformity parameters relating toxicity could not satisfy the RTOG/ABS recommendation (DHI $\geq 0.75$ ), which is stricter than that of the GEC-ESTRO (dose non-uniformity ratio $\geq 0.35$ ). However, our planning parameters had been provided based on both guidelines, with a strict avoidance of radiation exposure to the adjacent organs at risk (OARs) including the skin and the chest wall/ribs. This implied that the avoidance of hyperdose radiation to OARs was more relevant than the dose uniformity within PTV, because our target volume contained a large seroma as with an intracavitary brachytherapy technique. In fact, no radiation-induced toxicities such as telangiectasia and rib fracture [49] were observed in our cohort [18].

\section{Conclusions}

In CBD, additional anatomical information associated with implanted catheters and the cavity-to-CT/ clip-based seroma image could significantly reduce the LC volume and possibly the PTV for MIB-PBI planning. To our knowledge, CBD is a unique technique for an open-cavity catheter implant. A 3D-printed template has been reported as another reliable method to localize the target volume [50]. The LC delineated using this CBD technique is also reproducible and theoretically close to the true LC image, enabling the radiation oncologist to create more accurate LC with confidence. The real LC remains uncertain and the benefit of such a reduction to cosmetic outcomes is hardly proved. It may help to establish a standardized procedure in MIB-PBI and prevent unnecessary radiation exposure to the normal breast tissue, causing improvement in cosmetic outcomes.

\section{Acknowledgments}

We specify that this paper has not been published elsewhere. All authors have read and approved the content and agree to submit it for consideration of publication. We would also like to acknowledge with much appreciation the crucial role of Dr. Takayuki Nose, who gave his comments and technical advice.

\section{Disclosure}

Authors report no conflict of interest. 


\section{References}

1. Agarwal S, Pappas L, Neumayer L et al. Effect of breast conservation therapy vs mastectomy on disease-specific survival for early-stage breast cancer. JAMA Surg 2014; 149: 267-274.

2. Van Maaren MC, de Munck L, de Bock GH et al. 10-year survival after breast-conserving surgery plus radiotherapy compared with mastectomy in early breast cancer in the Netherlands: a population-based study. Lancet Oncol 2016; 17: 1158-1170.

3. Gu J, Groot G, Boden C et al. Review of factors influencing women's choice of mastectomy versus breast conserving therapy in early stage breast cancer: a systematic review. Clin Breast Cancer 2018; 18: e539-554.

4. Strnad V, Ott OJ. 5-year results of accelerated partial breast irradiation using sole interstitial multicatheter brachytherapy versus whole-breast irradiation with boost after breast-conserving surgery for low-risk invasive and in-situ carcinoma of the female breast: a randomised, phase 3, non-inferiority trial. Lancet 2016; 387: 229-238.

5. Polgár C, Ott OJ, Hildebrandt G et al. Late side-effects and cosmetic results of accelerated partial breast irradiation with interstitial brachytherapy versus whole-breast irradiation after breast-conserving surgery for low-risk invasive and in-situ carcinoma of the female breast: 5-year results of a randomised, controlled, phase 3 trial. Lancet Oncol 2017; 18: 259268.

6. Coles CE, Griffin CL, Kirby AM et al. Partial-breast radiotherapy after breast conservation surgery for patients with early breast cancer (UK IMPORT LOW trial): 5-year results from a multicentre, randomised, controlled, phase 3, noninferiority trial. Lancet 2017; 390: 1048-1060.

7. Peterson D, Truong PT, Parpia S et al. Predictors of adverse cosmetic outcome in the RAPID trial: an exploratory analysis. Int J Radiat Oncol Biol Phys 2015; 91: 968-976.

8. Wazer DE, Kaufman S, Cuttino L et al. Accelerated partial breast irradiation: an analysis of variables associated with late toxicity and long-term cosmetic outcome after highdose-rate interstitial brachytherapy. Int J Radiat Oncol Biol Phys 2006; 64: 489-495.

9. Schäfer R, Strnad V, Polgár C. Quality-of-life results for accelerated partial breast irradiation with interstitial brachytherapy versus whole-breast irradiation in early breast cancer after breast-conserving surgery (GEC-ESTRO): 5-year results of a randomised, phase 3 trial. Lancet Oncol 2018; 19: 834-844.

10. Jethwa KR, Kahila MM, Mara KC et al. Patient-reported outcomes of catheter-based accelerated partial breast brachytherapy and whole breast irradiation, a single institution experience. Breast Cancer Res Treat 2018; 169: 189-196.

11. Sato K, Fuchikami H, Kato M et al. Efficacy of single-stage breast-conserving treatment using multicatheter partial breast brachytherapy evaluated by GEC-ESTRO phase 3 trial. J Contemp Brachytherapy 2017; 9: 424-430.

12. Sato K, Fuchikami H, Takeda N et al. Validating the efficacy of single-stage breast-conserving therapy using multicatheter partial-breast brachytherapy based on updated ASTRO guidelines. J Radiat Res 2018; 59: 303-308.

13. Boersma LJ, Janssen T, Elkhuizen PH et al. Reducing interobserver variation of boost-CTV delineation in breast conserving radiation therapy using a pre-operative CT and delineation guidelines. Radiother Oncol 2012; 103: 178-182.

14. Krawczyk JJ, Engel B. The importance of surgical clips for adequate tangential beam planning in breast conserving surgery and irradiation. Int J Radiat Oncol Biol Phys 1999; 43: 347-350.

15. Yang TJ, Tao R, Elkhuizen PH et al. Tumor bed delineation for external beam accelerated partial breast irradiation: a systematic review. Radiother Oncol 2013; 108: 181-189.
16. Yang JD, Kim MC, Lee JW et al. Usefulness of oncoplastic volume replacement techniques after breast conserving surgery in small to moderate-sized breasts. Arch Plast Surg 2012; 39: 489-496.

17. Morrow M, Strom EA, Bassett LW et al. Standard for breast conservation therapy in the management of invasive breast carcinoma. CA Cancer J Clin 2002; 52: 277-300.

18. Sato K, Mizuno Y, Kato M et al. Intraoperative open-cavity implant for accelerated partial breast irradiation using highdose rate multicatheter brachytherapy in Japanese breast cancer patients: a single-institution registry study. J Cancer Ther 2012; 3: 822-830.

19. Major T, Gutiérrez C, Guix B et al. Recommendations from GEC ESTRO Breast Cancer Working Group (II): Target definition and target delineation for accelerated or boost partial breast irradiation using multicatheter interstitial brachytherapy after breast conserving open cavity surgery. Radiother Oncol 2016; 118: 199-204.

20. NSABP PROTOCOL B-39/RTOG PROTOCOL 0413. http:/ / rpc.mdanderson.org/rpc/credentialing/files/B39_Protocol1.pdf. Accessed on 10 Jul 2018

21. The Radiation Therapy Oncology Group. https://www. rtog.org/LinkClick.aspx?fileticket=SQhssxHu7Jg\%3d\&tabid=227 (accessed on 10 Jul 2018).

22. Smitt MC, Birdwell RL, Goffinet DR. Breast electron boost planning: comparison of CT and US. Radiology 2001; 219: 203-206.

23. Cochrane RA, Valasiadou P, Wilson AR et al. Cosmesis and satisfaction after breast-conserving surgery correlates with the percentage of breast volume excised. Br J Surg 2003; 90: 1505-1509.

24. Moran MS, Schnitt SJ, Giuliano AE et al. Society of Surgical Oncology-American Society for Radiation Oncology consensus guideline on margins for breast-conserving surgery with whole-breast irradiation in stages I and II invasive breast cancer. Int J Radiat Oncol Biol Phys 2014; 88: 553-564.

25. Immink JM, Putter H, Bartelink H et al. Long-term cosmetic changes after breast-conserving treatment of patients with stage I-II breast cancer and included in the EORTC 'boost versus no boost' trial. Ann Oncol 2012; 23: 2591-2598.

26. Den Hartogh MD, van den Bongard HJ, Davidson MT et al. Full-thickness closure in breast-conserving surgery: the impact on radiotherapy target definition for boost and partial breast irradiation. A multimodality image evaluation. Ann Surg Oncol 2014; 21: 3774-3779.

27. Landis DM, Luo W, Song J et al. Variability among breast radiation oncologists in delineation of the postsurgical lumpectomy cavity. Int J Radiat Oncol Biol Phys 2007; 67: 1299-1308.

28. Petersen RP, Truong PT, Kader HA et al. Target volume delineation for partial breast radiotherapy planning: clinical characteristics associated with low interobserver concordance. Int J Radiat Oncol Biol Phys 2007; 69: 41-48.

29. Maskarinec G, Nagata C, Shimizu H et al. Comparison of mammographic densities and their determinants in women from Japan and Hawaii. Int J Cancer 2002; 102: 29-33.

30. Rajaram N, Mariapun S, Eriksson M et al. Differences in mammographic density between Asian and Caucasian populations: a comparative analysis. Breast Cancer Res Treat 2017; 161: 353-362

31. Yang $\mathrm{Z}$, Chen J, Hu W et al. Planning the breast boost: how accurately do surgical clips represent the CT seroma? Radiother Oncol 2010; 97: 530-534.

32. Kass R, Kumar G, Klimberg VS et al. Clip migration in stereotactic biopsy. Am J Surg 2002; 184: 325-331.

33. Kirby AM, Yarnold JR, Evans PM et al. Tumor bed delineation for partial breast and breast boost radiotherapy planned in the prone position: what does MRI add to X-ray CT local- 
ization of titanium clips placed in the excision cavity wall? Int J Radiat Oncol Biol Phys 2009; 74: 1276-1282.

34. Mast M, Coerkamp E, Heijenbrok M et al. Target volume delineation in breast conserving radiotherapy: are co-registered CT and MR images of added value? Radiat Oncol 2014; 9: 65.

35. Genebes C, Chand ME, Gal J et al. Accelerated partial breast irradiation in the elderly: 5-year results of high-dose rate multi-catheter brachytherapy. Radiat Oncol 2014; 9: 115.

36. Cambeiro M, Martinez-Regueira F, Rodriguez-Spiteri N et al. Multicatheter breast implant during breast conservative surgery: Novel approach to deliver accelerated partial breast irradiation. Brachytherapy 2016; 15: 485-494.

37. Gurram L, Wadasadawala T, Joshi K et al. Multi-catheter interstitial brachytherapy for partial breast irradiation: an audit of implant quality based on dosimetric evaluation comparing intra-operative versus post-operative placement. J Contemp Brachytherapy 2016; 8: 116-121.

38. Cozzi S, Laplana M2, Najjari D et al. Advantages of intraoperative implant for interstitial brachytherapy for accelerated partial breast irradiation either frail patients with early-stage disease or in locally recurrent breast cancer. J Contemp Brachytherapy 2018; 10: 97-104.

39. Hieken TJ, Mutter RW, Jakub JW et al. A novel treatment schedule for rapid completion of surgery and radiation in early-stage breast cancer. Ann Surg Oncol 2016; 23: 3297-3303.

40. Aristei C, Maranzano E, Lancellotta V et al. Partial breast irradiation with interstitial multi-catheter high-dose-rate brachytherapy. Long-term results of a phase II prospective study. Radiother Oncol 2017; 124: 208-213.

41. Hannoun-Levi JM, Resch A, Gal J, Kauer-Dorner D. Accelerated partial breast irradiation with interstitial brachytherapy as second conservative treatment for ipsilateral breast tumour recurrence: multicentric study of the GEC-ESTRO Breast Cancer Working Group. Radiother Oncol 2013; 108: 226-231.

42. Sharma R, Spierer M, Mutyala S et al. Change in seroma volume during whole-breast radiation therapy. Int J Radiat Oncol Biol Phys 2009; 75: 89-93.

43. Vaidya JS, Wenz F, Bulsara M et al. Risk-adapted targeted intraoperative radiotherapy versus whole-breast radiotherapy for breast cancer: 5-year results for local control and overall survival from the TARGIT-A randomised trial. Lancet 2014; 383: 603-613.

44. Den Hartogh MD, van den Bongard HJ, Davidson MT et al. Full-thickness closure in breast-conserving surgery: the impact on radiotherapy target definition for boost and partial breast irradiation. A multimodality image evaluation. Ann Surg Oncol 2014; 21: 3774-3779.

45. Kasumi F, Takahashi K, Nishimura S et al. CIH-Tokyo experience with breast-conserving surgery without radiotherapy: 6.5-year follow-up results of 1462 patients. Breast J 2006; 12: S181-190.

46. Strnad V, Hannoun-Levi JM, Guinot JL et al. Recommendations from GEC -ESTRO Breast Cancer Working Group (I): Target definition and target delineation for accelerated or boost Partial Breast Irradiation using multicatheter interstitial brachytherapy after breast conserving closed cavity surgery. Radiother Oncol 2015; 115: 342-348.

47. Strnad V, Major T, Polgar C et al. ESTRO-ACROP guideline: Interstitial multi-catheter breast brachytherapy as Accelerated Partial Breast Irradiation alone or as boost - GEC-ESTRO Breast Cancer Working Group practical recommendations. Radiother Oncol 2018; 128: 411-420.

48. Hepel JT, Arthur D, Shaitelman S et al. American Brachytherapy Society consensus report for accelerated partial breast irradiation using interstitial multicatheter brachytherapy. Brachytherapy 2017; 16: 919-928.
49. Yoshida K, Otani Y, Nose T et al. Case report of a dose-volume histogram analysis of rib fracture after accelerated partial breast irradiation: interim analysis of a Japanese prospective multi-institutional feasibility study. J Contemp Brachytherapy 2018; 10: 274-278.

50. Aristei C, Lancellotta V, Piergentini M et al. Individualized 3D-printed templates for high-dose-rate interstitial multicathether brachytherapy in patients with breast cancer. Brachytherapy 2019; 18: 57-62. 